\title{
Evaluation of the finger wrinkling test: a pilot study
}

\author{
S. van Barneveld $\cdot$ J. van der Palen • \\ M. J. A. M. van Putten
}

Received: 10 January 2010/Accepted: 20 April 2010/Published online: 12 May 2010

(C) The Author(s) 2010. This article is published with open access at Springerlink.com

\begin{abstract}
Purpose Tilt table testing mainly evaluates the systemic cardiovascular part of the autonomic nervous system, while it is assumed that the finger wrinkling test assesses the peripheral part of the autonomic nervous system. In this study we explored whether the finger wrinkling test could be a useful test for autonomic dysfunction and whether the clinical evaluation of wrinkling can be improved by digital analysis of photographs.

Methods As much as 20 healthy subjects and 15 patients underwent tilt table testing and finger wrinkling testing. During the finger wrinkling test the right hand was immersed in water at $40^{\circ} \mathrm{C}$. The degree of wrinkling was assessed with a 5-point clinical scale at baseline, 5, 15 and $30 \mathrm{~min}$ of immersion. Photographs were taken at the same
\end{abstract}

S. van Barneveld $(\bowtie) \cdot$ M. J. A. M. van Putten

Department of Clinical Neurophysiology,

Medisch Spectrum Twente, Haaksbergerstraat 55,

7513 ER Enschede, The Netherlands

e-mail: sylvia_van_barneveld@hotmail.com

J. van der Palen

Department of Epidemiology, Medisch Spectrum Twente,

Enschede, The Netherlands

S. van Barneveld

Department of Medicine, University of Groningen,

Groningen, The Netherlands

M. J. A. M. van Putten

MIRA Institute for Biomedical Technology and Technical

Medicine, University of Twente, Enschede, The Netherlands

J. van der Palen

Department of Research Methodology, Measurement and Data Analysis, Faculty of Behavioral Sciences, University of Twente, Enschede, The Netherlands intervals. Several features were evaluated using digital analysis: length and gradient of automatically detected wrinkle and mean, maximum, minimum, variance and derivative of grey value of pixels.

Results Clinical scoring of wrinkling allowed differentiation between healthy subjects and patients with a normal and an abnormal response to tilt table testing. Relevant features obtained with digital analysis were mean grey value and the gradient of automatically detected wrinkle. McNemar's test showed no difference in test results between the tilt table test and the finger wrinkling test with a kappa of 0.68 .

Conclusion The finger wrinkling test can be used as a screening test before tilt table testing. Visual evaluation of wrinkling is still superior to digital analysis of photographs.

Keywords Autonomic dysfunction - Tilt table test .

Finger wrinkling test $\cdot$ Digital analysis

\section{Introduction}

In patients suffering from diabetes mellitus and hemiparkinsonism autonomic dysfunction is often present [1]. Diagnosis of autonomic dysfunction can be difficult to establish. There are some tests available, like tilt table testing, sympathetic skin response and sweat imprint test $[2,3]$. Sympathetic skin response and sweat imprint test evaluate the thermoregulatory part, while tilt table testing mainly evaluates the cardiovascular part of the autonomic nervous system [3]. Tilt table test is a simple, standardized test with a sensitivity of $75 \%$ and specificity of $97 \%$, when performed by Westminister protocol. Unfortunately reproducibility is poor [2]. Both sympathetic skin response and sweat imprint test have a poor sensitivity compared to 
tilt table testing and special equipment for testing is necessary [3].

The finger wrinkling test could be a complementary test to the above-mentioned tests, because one assumes that this test evaluates the autonomic nervous system of the limbs $[4,5]$. The main underlying mechanism of wrinkling during water immersion is vasoconstriction by sympathetic input [6]. During the finger wrinkling test a hand is immersed in water at $40^{\circ} \mathrm{C}$ for $30 \mathrm{~min}$ [5] and the amount of wrinkling is subsequently evaluated. The degree of wrinkling is assessed by a 5-point clinical scale [7] (see Table 1). However, this scale is a poor quantification method, because there is a wide variety of interpretation [4]. Previous studies have shown that the degree of wrinkling is less in patients with diabetes mellitus or hemiparkinsonism than in healthy subjects $[7,8]$. No correlation in the results between tilt table testing and finger wrinkling test has been evaluated [8] or found [7].

We explored whether the finger wrinkling test is a useful test for autonomic dysfunction in particular if there is a correlation between peripheral and systemic function of the autonomic nervous system as evaluated by tilt table testing. In addition, we investigated whether digital analysis of photographs of the hand could improve the quantification of wrinkling.

\section{Methods}

As much as 20 healthy subjects (10 men) were recruited from hospital staff and students. Their average age was 26 years (range 21-52 years). Exclusion criteria were cigarette smoking, skin disease, operation or trauma of the hand and use of medication that might interfere with autonomic function, such as beta blocking agents.

A total of 15 patients ( 8 men), who were referred for tilt table testing were included. Their average age was 58 years (range 21-78 years). Indications for referral were syncope $(n=8)$, dizziness $(n=2)$, diabetes mellitus $(n=2)$ and Parkinsonism $(n=3)$. All subjects gave informed consent and underwent tilt table testing and finger wrinkling testing. The study was conducted in accordance with the Helsinki Declaration and was approved by the Ethics

Table 1 Explanation of the 5-point clinical scale [7]

\begin{tabular}{ll}
\hline Grade & Description \\
\hline 0 & No evidence of any skin wrinkling at the fingertip \\
1 & The fingertip was not completely smooth \\
2 & Two or less lines of wrinkling on the fingertip \\
3 & Three or more lines of wrinkling on the fingertip \\
4 & Wrinkling completely distorting the pulp of the fingertip
\end{tabular}

Committee of Medisch Spectrum Twente, the Netherlands. Participants were instructed not to use any cream or lotion on their hands on the day of testing. Drinking coffee or tea $2 \mathrm{~h}$ prior to testing was not permitted.

During the tilt table test blood pressure, heart rate and ECG were recorded for $10 \mathrm{~min}$ in a horizontal position, $10 \mathrm{~min}$ in tilted position of $60^{\circ}$ and another $10 \mathrm{~min}$ in horizontal position. The result was considered abnormal if there were signs of orthostatic hypotension with a systolic drop of more than $20 \mathrm{mmHg}$ and diastolic drop of more than $10 \mathrm{mmHg}$ or/and if there was no compensatory heart rate acceleration more than $10-20 \mathrm{bpm}$ after tilting to $60^{\circ}$.

During the finger wrinkling test the right hand was immersed in a tempex bucket filled with water at $40^{\circ} \mathrm{C}$. Temperature was measured before and after the finger wrinkling test. Maximum decrease in temperature was $2^{\circ} \mathrm{C}$ over the total measurement period of $30 \mathrm{~min}$. The degree of wrinkling was assessed with the 5-point clinical scale at baseline, 5, 15 and $30 \mathrm{~min}$ of immersion. Only digiti 2-5 were judged, because it was difficult to make a good photograph of the thumb. Scores of digiti 2-5 were summed.

Photographs were taken at the same intervals. Before taking a photograph the right hand was held in front of a black background with the palm of the hand towards the digital camera (CANON D200), which was positioned at a distance of $94.5 \mathrm{~cm}$ of the black background. Diffuse lighting with BOWENS BW $3610^{\mathrm{TM}}$ was used on the right side of the right hand.

For digital analysis several features were calculated using Matlab (2007, The Mathworks Inc.): length and gradient of automatically detected wrinkles and mean, maximum, minimum, variance and derivative of grey values of the pixels. The result of digital analysis was considered abnormal if the value was below the cutoff point made by the ROC curve to determine optimal sensitivity and specificity. For evaluation of the different features of digital analysis the result of the finger wrinkling test was considered abnormal if there was no increase in score on the clinical scale or if the total score was less than 4 after finishing the finger wrinkling test.

\section{Statistics}

Mann-Whitney $U$ tests were used for comparison of clinical scale scores between healthy subjects and patients and comparisons between patients with a normal and abnormal tilt table result. To evaluate the features of digital analysis Student's $T$ test was used to analyse if there was a significant difference in value of the feature between healthy subjects and patients. Spearman's correlation coefficients were used to assess the degree of correlation between clinical scale scores and the different digital features 

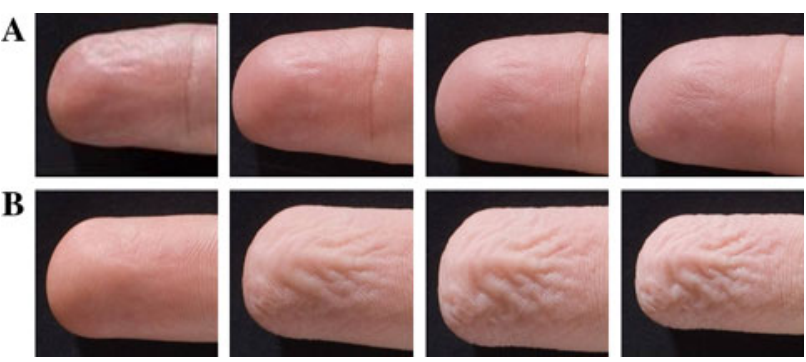

Fig. 1 The photographs are taken from left to right at $t=0,5,15$ and $30 \mathrm{~min}$. a Patient with an abnormal result on finger wrinkling test. Score at clinical scale at $t=30 \mathrm{~min}$ is 1 . b Result of a healthy subject. Score at clinical scale at $t=30 \mathrm{~min}$ is 4

Table 2 Values of the clinical wrinkling scale for healthy controls and patients

\begin{tabular}{lcclll}
\hline Time & \multicolumn{2}{l}{ Healthy subjects } & & \multicolumn{2}{l}{ Patients } \\
\cline { 2 - 3 } \cline { 5 - 6 } \cline { 5 - 6 } & Median & Interquartile range & & Median & Interquartile range \\
\hline 0 & 0 & $0-0$ & & 0 & $0-8$ \\
5 & 4 & $4-6.25$ & & 4 & $4-8$ \\
15 & 10 & $8-12$ & & 8 & $4-12$ \\
30 & 15 & $11.25-16$ & & 12 & $8-14$ \\
\hline
\end{tabular}

Median and interquartile range are used because of non-normal distribution. Scores of digiti 2-5 were summed

extracted. Sensitivity and specificity were determined with ROC curves. Mc Nemar's test was used to determine the correlation between the result of the finger wrinkling test and the tilt table test. With kappa the strength of the abovementioned relationship was analysed. Sensitivity and specificity for three cutoff values $(4,8$ and 12$)$ of the finger wrinkling test were determined with tilt table testing as gold standard.

\section{Results}

Figure 1 shows an example of photographs taken during the finger wrinkling test with a normal and an abnormal result. Clinical wrinkling scores are significantly higher for healthy subjects than for patients $(P<0.001)$. Also clinical scale scores are significantly higher for patients with a normal result of the tilt table test than patients with an abnormal result $(P=0.02)$ (Table 2$)$.
The features calculated for digital analysis have to meet the following criteria. First the features must show a different value for patients and for healthy subjects. Second the feature must correlate with the clinical scale $(r>0.30)$ with sufficient sensitivity and specificity. Two features met the above-mentioned criteria, namely mean of grey value and gradient of automatically detected wrinkle. The characteristics of these two features are shown in Table 3.

Sensitivity is $100 \%$ for mean of grey value, specificity is $97 \%$ for gradient of automatically detected wrinkle $(t=15$ to $t=0$ ) and there is a correlation of 0.77 with clinical scale score for gradient of automatically detected wrinkle $(t=30$ to $t=0)$.

As much as 28 persons had normal results on both tests, while 4 persons had abnormal results on both tests (Table 4). McNemar' $s$ test showed that there is no difference in test results between both tests $(P=1.00)$. Kappa of this relationship is 0.68 , which is considered good. When the finger wrinkling test is assessed by digital analysis the results are not so clear with kappa values between 0.19 and 0.30 for the two features with the best qualities.

Table 5 shows that a cutoff value of 4 has a sensitivity of $80 \%$ and specificity of $93 \%$. As cutoff values increase, sensitivity increases and specificity decreases.

\section{Discussion}

Our primary aim of this pilot study was to evaluate the finger wrinkling test for the evaluation of autonomic dysfunction. In addition, we explored if we could improve the clinical quantification of wrinkling by analysis of digital photographs.

We found a significant correlation between the results of the finger wrinkling test and tilt table testing, which indicates that patients with an abnormal result on the finger wrinkling test have a higher probability of having an abnormal result on the tilt table test than patients with a normal result on the finger wrinkling test.

This contrasts findings of Clark et al. [7], who found no correlation between the result of autonomic function tests and the degree of wrinkling in diabetics. Detailed comparison with this study is difficult, however, because the

Table 3 Overview of differentiating characteristics of two features

\begin{tabular}{lccc}
\hline Feature & Sensitivity & Specificity & Correlation with clinical scale \\
\hline Mean of grey values $t=30$ to $t=0$ & 100 & 72 & 0.34 \\
Gradient of detected wrinkle $t=15$ to $t=0$ & 67 & 97 & 0.50 \\
Gradient of detected wrinkle $t=30$ to $t=0$ & 83 & 76 & 0.77
\end{tabular}

Clinical scores on the finger wrinkling test were used as a gold standard 
Table 4 Correlation between the results of the tilt table test and the finger wrinkling test

\begin{tabular}{lll}
\hline Tilt table test & \multicolumn{2}{l}{ Finger wrinkling test } \\
\cline { 2 - 3 } & Normal & Abnormal \\
\hline Normal & 28 & 2 \\
Abnormal & 1 & 4 \\
\hline
\end{tabular}

Table 5 Three different cutoff values with their corresponding sensitivity and specificity

\begin{tabular}{lcl}
\hline Cutoff value & Sensitivity $(\%)$ & Specificity (\%) \\
\hline 4 & 80 & 93 \\
8 & 100 & 83 \\
12 & 100 & 60 \\
\hline
\end{tabular}

Gold standard is tilt table testing

study populations and methods are quite different [7]. Clark et al. only explored a correlation between autonomic function tests and wrinkling among the included diabetics. Also the result of the finger wrinkling was not defined in normal and abnormal.

Recent studies suggest that the finger wrinkling test evaluates the more distal portion of the autonomic nervous system [9, 10]. Teoh et al. [9] showed that the finger wrinkling test is a useful supportive test for diagnosing small fibre neuropathy. Wilder-Smith et al. [10] established that the finger wrinkling test can be used for predicting intraepidermal nerve fibre density. The correlation between the results of tilt table testing and finger wrinkling testing found in our pilot study suggests that in a significant fraction of patients both parts of the autonomic system are affected.

A striking result of this pilot study is the differentiation between healthy subjects and patients by clinical scores at baseline. There are some explanations for this finding. There were three patients with wrinkled hands at baseline. All these patients had abnormal results on tilt table test. Maybe wrinkled hands predict the outcome of the tilt table test. Also the average age was significantly higher for patients with abnormal results on tilt table test than other patients and healthy subjects.

The results of digital analysis of the photographs taken during the finger wrinkling test were disappointing. Only 2 out of the 11 evaluated features had differentiating characteristics. Most likely, the quality of the photographs was not sufficient to develop an accurate quantification method. The major problem was the illumination, which caused a strong shadow on the lower side of the fingers. We were not able to remove this shadow sufficiently, which interfered with the digital analysis of the amount of wrinkling.
As a first step in developing several features for analysis of digital photographs, our aim was to define features that differentiate between the presence and the absence of wrinkles. This motivated the use of a cutoff value of 4 since any wrinkle in a finger evaluated was considered abnormal. This contrast the cutoff value used by Teoh et al. [9], where a cutoff value of 8 was used. A second criterion for successful use of the digital analysis is that features must differentiate between various degrees of wrinkling. However, due to disappointing results obtained in the first step, as outlined above, we did not further pursue this approach.

Another problem of evaluating whether analysis of digital photographs is a better quantification method than the clinical evaluation is the lack of a gold standard. Although initially the sympathetic skin response (SSR) was proposed as potential gold standard [3], various studies have shown now that SSR is a poor autonomic function test. Vasudevan et al. [11] showed that this response did not have more advantages than the finger wrinkling test with equal sensitivity and specificity. Also Teoh et al. [9] reported that the sympathetic skin response was less useful in diagnosing small fibre neuropathy than the finger wrinkling test or intraepidermal nerve fibre density.

Given the limited specificity that we found in our comparison $(\sim 70 \%)$, abnormal results in finger wrinkling are not always due to dysfunction of the autonomic nervous system. For instance abnormal results could also be caused by smoking, trauma of the hand or medication interfering with the autonomic nervous system. These causes were not used as an exclusion criterion in patients, because we wished to investigate the practical relevance of the finger wrinkling test in a typical patient cohort referred for tilt table testing.

In summary, our pilot study suggests that the finger wrinkling test can be used as a screening test before tilt table testing. The assessment of wrinkling with the 5-point clinical scale is still superior to digital analysis.

Acknowledgments We would like to thank Mr. R. Elsjan, Mr. S. van der Meer and Ms. M. Lenselink of our hospital for their advices and their photograph material. Also Prof. C.H. Slump, Mr. D.J. Kroon, Mrs. M. Heijblom, Mrs. M.C. Cloostermans, Mrs. L. Melching and Mrs. S. Sharifi are acknowledged for their contribution to the technical aspects of this pilot study. The assistance of the technicians of the department of clinical neurophysiology is also highly appreciated.

Conflict of interest statement The authors declare that they have no conflict of interest.

Open Access This article is distributed under the terms of the Creative Commons Attribution Noncommercial License which permits any noncommercial use, distribution, and reproduction in any medium, provided the original author(s) and source are credited. 


\section{References}

1. Donofrio PD, Caress JB (2001) Autonomic disorders. Neurologist 7:220-233

2. Brignole M, Alboni P, Benditt DG et al (2004) Guidelines on management (diagnosis and treatment) of syncope. Update 2004. Europace 6:467-537

3. Ravits JM (1997) AAEM Minimonograph \#48: autonomic nervous system testing. Muscle Nerve 20:919-937

4. Wilder-Smith EPV (2004) Water immersion wrinkling physiology and use as an indicator of sympathetic function. Clin Auton Res 14:125-131

5. Bull C, Henry JA (1977) Finger wrinkling as a test of autonomic function. Br Med J 1:551-552

6. Wilder-Smith EPV, Chow A (2003) Water immersion wrinkling is due to vasoconstriction. Muscle Nerve 27:307-311
7. Clark CV, Pentland B, Ewing DJ, Clarke BF (1984) Decreased skin wrinkling in diabetes mellitus. Diabetes Care 7:224-227

8. Djaldetti R, Melamed E, Gadoth N (2001) Abnormal skin wrinkling in the less affected side in hemiParkinsonism-a possible test for sympathetic dysfunction in Parkinson's disease. Biomed Pharmacother 55:475-478

9. Teoh HL, Chow A, Wilder-Smith EPV (2008) Skin wrinkling for diagnosing small fibre neuropathy: comparison with epidermal nerve density and sympathetic skin response. J Neurol Neurosurg Psychiatry 79:835-837

10. Wilder-Smith EPV, Guo Y, Chow A (2009) Stimulated skin wrinkling for predicting intraepidermal nerve fibre density. J Clin Neurophysiol 120:953-958

11. Vasudevan TM, van Rij AM, Nukada H, Taylor PK (2000) Skin wrinkling for the assessment of sympathetic function in the limbs. Aust NZ J Surg 70:57-59 